Pacific Journal of Mathematics

NORMAL SUBGROUPS OF SOME HOMEOMORPHISM 


\section{NORMAL SUBGROUPS OF SOME HOMEOMORPHISM GROUPS*}

\section{J. V. WHITTAKER}

1. Introduction. The normal subgroups of the group of all homeomorphisms of a space $X$ have been enumerated by Fine and Schweigert [2] when $X$ is a line, by Schreier and Ulam [3] when $X$ is a circle, by Ulam and von Neumann [4] and Anderson [1] when $X$ is a 2-sphere. In each of these cases there are either one or two proper normal subgroups. However, when $X$ is an $n$-cell $(n>1)$, there are infinitely many. The object of this paper is to investigate the normal subgroups for a class of spaces $X$ which includes the $n$-cell. Some of these normal subgroups, although not all, can be defined in terms of the family of fixed point sets of their elements, and we investigate this relationship at some length. A smallest normal subgroup is exhibited, and the corresponding quotient group is represented as a group of transformations of a related space.

2. Families of fixed point sets. Let $X$ be a set, $\Pi(X)$ the group of all permutations of $X$ (one-to-one mappings of $X$ onto itself), and $G$ a subgroup of $\Pi(X)$. Suppose that $\mathscr{F}$ is a non-empty family of subsets of $X$ satisfying the following conditions:

(i) If $F_{1}, F_{2} \in \mathscr{F}$, then there exists an $F_{3} \in \mathscr{F}$ such that $F_{3} \subset$ $F_{1} \cap F_{2}$,

(ii) If $F_{1} \in \mathscr{F}$ and $g \in G$, then there exists an $F_{2} \in \mathscr{F}$ such that $F_{2} \subset g\left(F_{1}\right)$.

We shall call $\mathscr{F}$ ecliptic relative to $G$. For example, if $\mathscr{F}$ consists of the complements of all finite subsets of $X$, then $\mathscr{F}$ is ecliptic relative to $\Pi(X)$. If $X$ has a topology, we denote the group of homeomorphisms of $X$ by $H(X)$. Let $X$ be a closed unit ball $B_{n}$ in euclidean $n$-space and $\mathscr{F}_{0}$ consist of the complements in $B_{n}$ of those balls which are concentric with $B_{n}$ and have radius less than one. Then $\mathscr{F}_{0}$ is ecliptic relative to $H\left(B_{n}\right)$. In this connection, we note that for $h \in$ $H\left(B_{n}\right), h\left(S_{n-1}\right)=S_{n-1}$, where $S_{n-1}$ is the boundary of $B_{n}$.

Let $X$ again be an arbitrary set and $G$ a subgroup of $\Pi(X)$. We introduce a partial ordering among the families of subsets of $X$ as follows: $\mathscr{F} \leqq \mathscr{F}^{\prime}$ provided that, for every $F \in \mathscr{F}$, there exists an $F^{\prime} \in$ $\mathscr{F}^{\prime}$ such that $F^{\prime} \subset F$. Evidently $\mathscr{F} \subset \mathscr{F}^{\prime}$ implies $\mathscr{F} \leqq \mathscr{F}^{\prime}$, where $\mathscr{F} \subset \mathscr{F}^{\prime}$ means set inclusion, but the converse is false. We define equivalence of $\mathscr{F}$ and $\mathscr{F}^{\prime}$ to mean $\mathscr{F}^{-} \leqq \mathscr{F}^{\prime}$ and $\mathscr{F}^{\prime} \leqq \mathscr{F}$, and we write $\mathscr{F} \cong \mathscr{F}^{\prime}$.

Received October 26, 1959. This paper was written while the author was a Summer Research Institute Fellow of the Canadian Mathematical Congress. 
Lemma 1. If $\mathscr{F}, \mathscr{F}^{\prime}$ are families of subsets of $X, \mathscr{F}^{\prime} \cong \mathscr{F}^{\prime}$, and $\mathscr{F}^{-}$is ecliptic relative to $G$, then $\mathscr{F}^{\prime}$ is ecliptic relative to $G$.

Proof. If $F_{1}^{\prime}, F_{2}^{\prime} \in \mathscr{F}^{\prime}$, then there exist sets $F_{1}, F_{2}, F_{3} \in \mathscr{F}$ and $F_{3}^{\prime} \in \mathscr{F}^{\prime}$ such that $F_{1} \subset F_{1}^{\prime}, F_{2} \subset F_{2}^{\prime}$, and $F_{3}^{\prime} \subset F_{3} \subset F_{1} \cap F_{2} \subset F_{1}^{\prime} \cap F_{2}^{\prime}$. Second, if $F_{1}^{\prime} \in \mathscr{F}^{\prime}$ and $g \in G$, then we can find $F_{1}, F_{2} \in \mathscr{F}^{-}$and $F_{2}^{\prime} \in$ $\mathscr{F}^{\prime}$ such that $F_{1} \subset F_{1}^{\prime}$ and $F_{2}^{\prime} \subset F_{2} \subset g\left(F_{1}\right) \subset g\left(F_{1}^{\prime}\right)$.

To any family $\mathscr{F}$ we can adjoin all subsets of $X$ which contain some element of $\mathscr{F}$ and thus obtain a family $\mathscr{F}^{*}$ which is clearly equivalent to $\mathscr{F}$ and, by Lemma 1 , is ecliptic relative to $G$ if $\mathscr{F}$ is. In fact, $\mathscr{F}^{*}$ has the property that $F_{1}^{*}, F_{2}^{*} \in \mathscr{F}^{*}$ and $g \in G$ implies $F_{1}^{*} \cap F_{2}^{*}, g\left(F_{1}^{*}\right) \in \mathscr{F}^{*}$. In addition, $\mathscr{F}^{*}$ is an upper bound, with respect to set inclusion, among the families equivalent to $\mathscr{F}$. We shall call $\mathscr{F}$ replete if it is equivalent to no larger family.

If $f \in \Pi(X)$, we set $K(f)=\{x \in X: f(x)=x\}$. For any family $\mathscr{F}$ of subsets of $X$, we define

$$
S(\mathscr{F}, G)=\{g \in G: K(g) \supset F \text { for some } F \in \mathscr{F}\} \text {. }
$$

We note that if the empty set $\varnothing \in \mathscr{F}$, then $S(\mathscr{F}, G)=G$.

Lemma 2. (a) $\mathscr{F} \cong \mathscr{F}^{\prime}$ implies $S(\mathscr{F}, G)=S\left(\mathscr{F}^{\prime}, G\right)$.

(b) If $\mathscr{F}$ satisfies (i), then $S(\mathscr{F}, G)$ is a subgroup of $G$.

(c) If $f \in \Pi(X)$, then

$$
f[S(\mathscr{F}, G)] f^{-1}=S\left(f(\mathscr{F}), f G f^{-1}\right) .
$$

(d) If $\mathscr{F}$ is ecliptic relative to $G$, then $S(\mathscr{F}, G)$ is a normal subgroup of $G$.

Proof. For (a) we show that $\mathscr{F} \leqq \mathscr{F}^{\prime}$ implies $S(\mathscr{F}, G) \subset S\left(\mathscr{F}^{\prime}, G\right)$. Indeed, if $g \in S(\mathscr{F}, G)$ and $K(g) \supset F$ for some $F \in \mathscr{F}$, we can find $F^{\prime} \in \mathscr{F}^{\prime}$ such that $F^{\prime} \subset F \subset K(g)$, whence $g \in S\left(\mathscr{F}^{\prime}, G\right)$. In (b) we need merely observe that, for any $f_{1}, f_{2} \in \Pi(X), K\left(f_{1}, f_{2}\right) \supset K\left(f_{1}\right) \cap K\left(f_{2}\right)$ and $K\left(f_{1}^{-1}\right)=K\left(f_{1}\right)$. In part (c) we use the relation $K\left(f g f^{-1}\right)=f(K(g))$. If $g \in f[S(\mathscr{F}, G)] f^{-1}$, then $g=f g_{1} f^{-1}$, where $g_{1} \in G$ and $K\left(g_{1}\right) \supset F$ for some $F \in \mathscr{F}$. Hence, $g \in f G f^{-1}, K(g) \supset f(F)$, and $g \in S\left(f(\mathscr{F}), f G f^{-1}\right)$. If $g \in S\left(f(\mathscr{F}), f G f^{-1}\right)$, then $g=f g_{1} f^{-1}$ for some $g_{1} \in G$, and $K(g) \supset f(F)$ for some $F \in \mathscr{F}$. Hence, $K\left(g_{1}\right) \supset F$, and $g \in f[S(\mathscr{F}, G)] f^{-1}$. In part (d), let $f \in G$. From (c), $f[S(\mathscr{F}, G)] f^{-1}=S(f(\mathscr{F}), G)$. Normality will follow from (a) if we can show that $f(\mathscr{F}) \cong \mathscr{F}$. Clearly (ii) implies $f(\mathscr{F}) \leqq \mathscr{F}$. If $F_{1} \in \mathscr{F}$, then there is an $F_{2} \in \mathscr{F}$ such that $F_{2} \subset f^{-1}\left(F_{1}\right)$, whence $f\left(F_{2}\right) \subset F_{1}$, and $\mathscr{F} \leqq f(\mathscr{F})$.

We shall assume, from now on, that $X$ is a Hausdorff topological space, unless the contrary is explicitly stated. For $S(\mathscr{F}, H(X))$ we shall 
write $S(\mathscr{F})$, and if $\mathscr{F}$ is ecliptic relative to $H(X)$, we shall simply say that $\mathscr{F}$ is ecliptic. For any family of subsets of $X$, we introduce a further condition:

(iii) If $F \in \mathscr{F}$ and $U \subset X$ is open $(U \neq \varnothing)$, then there exists an $h \in H(X)$ such that $h(c F) \subset U$, where $c F$ is the complement of $F$ in $X$. An ecliptic family which satisfies (iii) will be called strictly ecliptic. The family $\mathscr{F}_{0}$ of subsets of $B_{n}$ defined above is evidently strictly ecliptic. If $\mathscr{F}$ satisfies (iii) and $\mathscr{F} \geqq \mathscr{F}^{\prime}$, then clearly $\mathscr{F}^{\prime}$ satisfies (iii). Since $K(h)$ is closed for every $h \in H(X)$, there is no loss of generality in assuming that the elements of any family $\mathscr{F}$ are closed, and this assumption will be made from now on, unless the contrary is stated.

Lemma 3. If $X$ admits families $\mathscr{F}, \mathscr{F}^{\prime}$ which satisfy (ii) and (iii) and contain more than one element, then $\mathscr{F} \cong \mathscr{F} '$.

Proof. We may as well assume that $\mathscr{F}_{\mathscr{F}} \mathscr{F}^{\prime}$ are replete in the closed subsets of $X$. If $F \in \mathscr{F}, F \neq X$, and $F^{\prime} \in \mathscr{F}^{\prime}$, then we can find $h \in H(X)$ such that $h\left(c F^{\prime}\right) \subset c F$. Hence, $h\left(F^{\prime}\right) \supset F, h\left(F^{\prime}\right) \in \mathscr{F}$, and $F^{\prime}=$ $h^{-1}\left(h\left(F^{\prime}\right)\right) \in \mathscr{F}$. Thus $\mathscr{F}^{\prime} \subset \mathscr{F}$ and, similarly $\mathscr{F} \subset \mathscr{F}^{\prime}$.

Some spaces contain no ecliptic families except $\{X\}$ and the set $\mathscr{C}(X)$ of all closed subsets of $X$. For, by Lemma 2, such a family defines a normal subgroup of $H(X)$; when $X$ is a 1-sphere, Schreier and Ulam [3] showed that the only proper normal subgroup of $H(X)$ consists of the orientation-preserving elements of which some have no fixed points.

3. Minimal normal subgroups. We shall need to know something more about $H(X)$. Rather than make specific and detailed assumptions about the existence of certain homeomorphisms, we shall assume a mildly euclidean structure for $X$, namely:

(iv) If $U \subset X$ is open $(U \neq \varnothing)$, then there exists an open $V \subset U$ which is homeomorphic to an open ball in a euclidean space of positive dimension.

The dimension of the ball may vary for different open sets. We shall refer to $V$ as a euclidean neighborhood in $X$.

TheOREM 1. Suppose $X$ satisfies (iv) and contains a strictly ecliptic family $\mathscr{F}$. If $N$ is a normal subgroup of $H(X)$, then either $N \supset$ $S(\mathscr{F})$ or $N$ consists of the identity $e$.

Proof. Suppose $N \neq\{e\}$ and $g_{0} \in N, g_{0} \neq e$. Then $g_{0}(x) \neq x$ for some $x \in X$, and we can find a neighborhood $U_{0}$ of $x$ and a euclidean neighborhood $V_{0}$ such that $g_{0}\left(U_{0}\right) \cap U_{0}=\varnothing$ and $V_{0} \subset g_{0}\left(U_{0}\right)$. Let $\omega$ map $V_{0}$ homeomorphically onto an open ball in some euclidean space, let $B \subset \omega\left(V_{0}\right)$ be a closed unit ball of the same dimension, and set $W_{0}=\omega^{-1}$ (int $B$ ), where 
int denotes interior. We wish to construct a homeomorphism $h_{0}$ of $\bar{W}_{0}$ in its relative topology with the following properties:

(a) $K\left(h_{0}\right) \supset \bar{W}_{0} \cap c W_{0}$,

(b) there exists an open $V \subset W_{0}$ such that, for all integers $n>0$, $h_{0}^{n}(\bar{V}) \cap \bar{V}=\varnothing$,

(c) if $A=\bigcup_{n=0}^{\infty} h_{0}^{n}(\bar{V})$, then $\bar{A} \cap c A$ is a single point. To do this is evidently equivalent to constructing such a homeomorphism $k_{0}$ of $B$, for then $h_{0}=\omega^{-1} k_{0} \omega$ has the desired properties in $\bar{W}_{0}$. Let $\theta$ be a homeomorphism of $[0,1]$ such that $K(\theta)=\{0,1\}$ and $\theta(r)<r$ for $0<r<1$. If $p \in B$ lies at a distance $r$ from the center of $B$, then we define $k_{0}(p)$ to be the point on the same radial line at a distance $\theta(r)$ from the center. By choosing a sufficiently small open ball in $B$ which does not meet either the center or boundary, we can satisfy (a), (b), and (c).

We now define the function $h_{1}$ as follows: $h_{1}(x)=h_{0}(x)$ if $x \in W_{0}$, $h_{1}(x)=x$ if $x \in c W_{0}$ Clearly, $h_{1} \in H(X)$. Now $g_{1}=g_{0} h_{1}^{-1} g_{0}^{-1} h_{1} \in N$ since $N$ is normal, and $g_{0} h_{1}^{-1} g_{0}^{-1} h_{1}(x)=h_{1}(x)$ for $x \in W_{0}$, since $g_{0}^{-1}\left(W_{0}\right) \subset c W_{0}$. Thus $g_{1}(x)=h_{0}(x)$ for $x \in W_{0}$. Let $g$ be any element of $S(\mathscr{F})$. Then there exists an $F \in \mathscr{F}$ and $h_{2} \in H(X)$ such that $K(g) \supset F$ and $h_{2}(c F) \subset V$. Thus $K\left(h_{2} g h_{2}^{-1}\right) \supset c V$. If we can construct an $h \in H(X)$ such that

$$
g_{1}^{-1} h g_{1} h^{-1}=h_{2} g h_{2}^{-1}=f,
$$

then we will have shown that $g \in N$ and $S(\mathscr{F}) \subset N$, since the left member of (1) lies in $N$. Let us rewrite (1) as $h g_{1}=g_{1} f h$. We set

$$
h(x)=\left\{\begin{array}{l}
g_{1}^{n} f g_{1}^{-n}(x) \quad \text { for } \quad x \in g_{1}^{n}(V), \quad n=1,2, \cdots, \\
x \text { for } x \in c\left(\bigcup_{n=1}^{\infty} g_{1}^{n}(V)\right) .
\end{array}\right.
$$

By property (b) above, $m \neq n$ implies $g_{1}^{m}(V) \cap g_{1}^{n}(V)=\varnothing$, whence $h$ is single-valued. Since $K(f) \supset c V$, the restriction of $f$ to $\bar{V}$ is a homeomorphism of $\bar{V}$, and the same holds for $g_{1}^{n} f g_{1}^{-n}$ and $g_{1}^{n}(\bar{V}), n=1,2, \cdots$. Let $\bar{A} \cap c A$ consist of the point $x_{0}$, where $A=\bigcup_{n=0}^{\infty} g_{1}^{n}(\bar{V})$. Then each $x \neq x_{0}$ has a neighborhood which meets at most one of the sets $g_{1}^{n}(\bar{V})$, and $h, h^{-1}$ are evidently continuous at such points. By the construction of $h_{0}$ and $V$, every neighborhood of $x_{0}$ contains all but a finite number of the sets $g_{1}^{n}(\bar{V})$, whence $h, h^{-1}$ are continuous here as well. Hence, $h \in H(X)$. If $x \in c \bar{A}$, then $g_{1}(x) \in K(h)$ and $h g_{1}(x)=g_{1} f h(x)$. When $x \in V$,

$$
h g_{1}(x)=g_{1} f g_{1}^{-1}\left(g_{1}(x)\right)=g_{1} f(x)=g_{1} f h(x) \text {. }
$$

Finally, if $n \geqq 1$ and $x \in g_{1}^{n}(V)$, we have $g_{1}^{n}(V) \subset K(f)$, so that $g_{1} f g_{1}^{n}(y)=$ $g_{1}^{n+1}(y)$ when $y \in V$. Hence,

$$
h g_{1}(x)=g_{1}^{n+1} f g_{1}^{-n-1}\left(g_{1}(x)\right)=g_{1} f g_{1}^{n} f g_{1}^{-n}(x)=g_{1} f h(x) .
$$

This establishes (1) and completes the proof. 
We offer the following example of a non-Hausdorff space $X$ without euclidean neighborhoods which admits a strictly ecliptic family $\mathscr{F}$ such that $S(\mathscr{F})$ is not minimal. Let $X$ be an infinite set in which $\mathscr{C}(X)$ consists of the finite subsets of $X$ and $X$ itself. Then $H(X)=\Pi(X)$. For $\mathscr{F}$ we take the collection of non-empty open sets and form $S(\mathscr{F})$. Since $X$ is not Hausdorff, $K(h)$ need not be closed for $h \in H(X)$. Clearly $\mathscr{F}$ is strictly ecliptic, but $S(\mathscr{F})$ contains, as a proper normal subgroup, the set of $h \in H(X)$ such that $c K(h)$ is finite and $h$ is an even permutation of $c K(h)$.

4. Normal subgroups of $H\left(B_{n}\right)$. As we remarked in $\S 2$, the family $\mathscr{F}_{0}$ of complements of smaller, open, concentric balls in $B_{n}$ is strictly ecliptic. When $\mathscr{F}_{0}$ is extended to a replete family, it will consist of all closed sets containing a neighborhood of the boundary $S_{n-1}$. In this section, we will also be concerned with the group $H_{0}\left(B_{n}\right)$ of those $h \in H\left(B_{n}\right)$ such that $K(h) \supset S_{n-1}$. Evidently $H_{0}\left(B_{n}\right) \supset S\left(\mathscr{F}_{0}\right)$, and $H_{0}\left(B_{n}\right)$ is normal in $H\left(B_{n}\right)$.

THEOREM 2. If $N$ is a normal subgroup of $H\left(B_{n}\right)$ which contains an element not in $H_{0}\left(B_{n}\right)$, then $N \supset H_{0}\left(B_{n}\right)$.

Proof. We will assume, to begin with, than $n \geqq 2$. Suppose $g_{0} \in$ $N \cap c H_{0}\left(B_{n}\right)$, and choose $x \in S_{n-1}$ so that $g_{0}(x) \neq x$. Let $W_{0}$ be the part of an open ball with center $x$ which lies in $B_{n}$ and is small enough so that $g_{0}\left(W_{0}\right) \cap W_{0}=\varnothing$. We wish to construct a homeomorphism $h_{0}$ of $\bar{W}_{0}$ and an open set $W \subset W_{0}$ such that $W \cap S_{n-1} \neq \varnothing$ and $h_{0}, W$ satisfy (a), (b), (c) in the proof of Theorem 1. Let $B, k_{0}$, and $V$ be the same as in that proof. If $I$ is an $(n-1)$-dimensional hyperplane which passes through the center of $B$ and meets $V$, then $I$ divides $B$ into two regions (including boundaries) $\Delta, \Delta^{\prime}$ such that $\Delta \cap \Delta^{\prime}=\Pi$. The restriction of $k_{0}$ to $\Delta$ is evidently a homeomorphism of $\Delta$. Let $\psi$ map $\Delta$ homeomorphically onto $\bar{W}_{0}$ in such a way that $\psi(\Pi)=\bar{W}_{0} \cap S_{n-1}$. Then $h_{0}=\psi k_{0} \psi^{-1}$ and $W=\psi(\Delta \cap V)$ clearly satisfy (a), (b), (c). We define $h_{1}(x)=h_{0}(x)$ for $x \in$ $W_{0}, h_{1}(x)=x$ for $x \in c W_{0}$, so that $h_{1} \in H\left(B_{n}\right)$. Then $g_{1}=g_{0} h_{1}^{-1} g_{0}^{-1} h_{1} \in N$, and $g_{1}(x)=h_{1}(x)$ for $x \in W_{0}$, as in the proof of Theorem 1. If $g$ is any element of $H_{0}\left(B_{n}\right)$ such that $K(g) \supset c W$, it follows from the construction in the same proof that $g \in N$.

Let $p, q \in S_{n-1}$ be antipodal, $D$ the diameter joining them, and $\Pi_{1}$, $\Pi_{2} \subset B_{n}$ two $(n-1)$-dimensional hyperplanes perpendicular to $D$. Now $\Pi_{1}, \Pi_{2}$ divide $B_{n}$ into three regions (including boundaries) $\Delta_{1}, \Delta_{2}, \Delta_{3}$ and, correspondingly, $S_{n-1}$ into three zones (including boundaries) $Z_{1}, Z_{2}, Z_{3}$. We take $\Delta_{2}$ to be the middle region, so that $\Delta_{1} \cap \Delta_{2}=\Pi_{1}, \Delta_{2} \cap \Delta_{3}=\Pi_{2}$, $p \in \Delta_{1}, q \in \Delta_{3}$. Let $P, Q$ be arbitrary neighborhoods of $p, q$, respectively, such that $\bar{P} \subset \dot{\Delta}_{1}, \bar{Q} \subset \Delta_{3}$.

Next, we construct $h_{2}, h_{3} \in H\left(B_{n}\right)$ such that $h_{2}(\bar{P}) \supset c W, h_{3}(\bar{Q}) \supset c W$. 
For example, $h_{2}$ might first expand $\bar{P}$ until its complement is quite small and then rotate the complement into $W$. If $g \in H_{0}\left(B_{n}\right)$ and $K(g) \supset \bar{P}$, then $K\left(h_{2} g h_{2}^{-1}\right) \supset c W$, whence $g \in N$. Similarly, $K(g) \supset \bar{Q}$ implies $g \in N$. We now wish to construct a homeomorphic mapping $\theta$ of $B_{n}$ onto $\Delta_{2} \cup \Delta_{3}$ such that $\theta(x)=x$ for all $x \in \Delta_{3}$. To accomplish this, we introduce spherical coordinates $r, \phi_{1}, \cdots, \phi_{n-1}$ for the points $x \in B_{n}$ such that $\phi_{1}$ is the angle between $D$ and the radial line through $x$. Then $\Pi_{i}$ satisfies the equation $r \cos \phi_{1}=k_{i},\left|k_{i}\right|<1(i=1,2)$. Let $r, \phi_{1}$ be regarded as polar coordinates for the closed upper half-plane in euclidean 2-space, and let $R$ be the set of $\left(r, \phi_{1}\right)$ such that $r \leqq 1,0 \leqq \phi_{1} \leqq \pi$. The lines $r \cos$ $\phi_{1}=k_{i}(i=1,2)$ divide $R$ into three regions (including boundaries) $R_{1}$, $R_{2}, R_{3}$. Let $\omega$ be a homeomorphic mapping of $R$ onto $R_{2} \cup R_{3}$ such that $\omega(y)=y$ for all $y \in R_{3}$. We then set

$$
\theta\left(r, \phi_{1}, \cdots, \phi_{n-1}\right)=\left(\omega\left(r, \phi_{1}\right), \phi_{2}, \cdots, \phi_{n-1}\right) .
$$

Let $f$ be any element of $H_{0}\left(B_{n}\right)$. Then $\theta f \theta^{-1} \in H\left(\Delta_{2} \cup \Delta_{3}\right)$, and $K\left(\theta f \theta^{-1}\right) \supset I_{1} \cup Z_{2} \cup Z_{3}$. We define $g_{2}(x)=\theta f \theta^{-1}(x)$ if $x \in \Delta_{2} \cup \Delta_{3}, g_{2}(x)=x$ if $x \in \Delta_{1}$. Clearly, $g_{2} \in H\left(B_{n}\right)$, and $K\left(g_{2}\right) \supset \bar{P}$, whence $g_{2} \in N$. In addition, $g_{2}(x)=f(x)$ for $x \in \Delta_{3} \cap f^{-1}\left(\Delta_{3}\right)$, so that $K\left(g_{2}^{-1} f\right) \supset \bar{Q}=\Delta_{3} \cap f^{-1}\left(\Delta_{3}\right)$, and $g_{3}=$ $g_{2}^{-1} f \in N$. Hence, $f=g_{2} g_{3} \in N$, and $H_{0}\left(B_{n}\right) \subset N$. When $n=1$, the constructions in the first half of the proof can not be carried out in $S_{0}$. The theorem follows, in this case, from the result obtained in [2] that the only proper normal subgroups of $H\left(B_{1}\right)$ are $S\left(\mathscr{F}_{0}\right)$ and $H_{0}\left(B_{1}\right)$.

If $G \subset \Pi(X)$ and $Y \subset X$, we denote the restrictions of the elements of $G$ to $Y$ by $G \mid Y$. For any orientable space $X$, we let $E(X)$ denote the group of all orientation-preserving homeomorphisms of $X$.

Lemma 4. If $N$ is a normal subgroup of $H\left(B_{n}\right)$, then $N \mid S_{n-1}$ is a normal subgroup of $H\left(S_{n-1}\right)$.

Proof. Clearly $N \mid S_{n-1}$ is a subgroup of $H\left(S_{n-1}\right)$. If $h_{0} \in H\left(S_{n-1}\right)$, we can extend $h_{0}$ to an element $h$ of $H\left(B_{n}\right)$. Let $p_{0}$ be the center of $B_{n}, p \neq p_{0}$ a point of $B_{n}$ lying on the sphere $S$ with center $p_{0}$, and $\pi$ the radial projection of $S$ onto $S_{n-1}$. We define $h(p)=\pi^{-1} h_{0} \pi(p), h\left(p_{0}\right)=p_{0}$. Clearly $h \in H\left(B_{n}\right)$. Then $N\left|S_{n-1}=\left(h N h^{-1}\right)\right| S_{n-1}=h_{0}\left(N \mid S_{n-1}\right) h_{0}^{-1}$.

Corollary. If $N$ is not contained in $H_{0}\left(B_{n}\right)$ and $n \leqq 3$, then $N$ is either $E\left(B_{n}\right)$ or $H\left(B_{n}\right)$.

Proof. By Lemma 4, N|S $S_{n-1}$ is a normal subgroup of $H\left(S_{n-1}\right)$ different from $\{e\}$. It was proved in [3] for $n=2$ and in [1] for $n=3$ that the only normal subgroups of $H\left(S_{n-1}\right)$ are $\{e\}, E\left(S_{n-1}\right)$, and $H\left(S_{n-1}\right)$. Hence, if $h \in E\left(B_{n}\right)$, there exists a $g \in N$ such that $h\left|S_{n-1}=g\right| S_{n-1}$. 
Then $f=g^{-1} h \in H_{0}\left(B_{n}\right) \subset N$ by Theorem 2, and $h=g f \in N$. If $N \not \subset E\left(B_{n}\right)$, a similar argument shows that $N=H\left(B_{n}\right)$. We note that $H_{0}\left(B_{1}\right)=E\left(B_{1}\right)$.

5. The lattice of normal subgroups. In the first part of this section, we revert to the assumption that $X$ is an arbitrary set. The intersection of two ecliptic families may be empty. If, for example, . $\mathscr{F}_{0}$ is the ecliptic family defined above for $B_{2}$ and $\mathscr{T}_{1}$ is the family of complements of interiors of simple polygons lying entirely in the interior of $B_{2}$, then $\mathscr{F}_{0} \cap \mathscr{F}_{1}=\varnothing$, although $\mathscr{F}_{0}^{*}=\mathscr{F}_{1}^{*}$. However, the intersection of any collection of replete, ecliptic families is also replete, ecliptic, and non-empty, since it always contains $\{X\}$. The smallest ecliptic family (up to equivalence) which contains a given collection $\left\{\mathscr{F}_{\alpha}\right\}$ of ecliptic families consists of all finite intersections of elements in $\cup \mathscr{F}_{\alpha}$. We denote this set by $\vee \mathscr{F}_{\alpha}$ and set $\wedge \mathscr{F}_{\alpha}=\cap \mathscr{F}_{\alpha}$. If the $\mathscr{F}_{\alpha}$ are replete, then $\vee \mathscr{F}_{\alpha}$ is also replete. For if $F_{1}, \cdots, F_{n} \in \cup \mathscr{F}_{\alpha}$, and $F \supset \bigcap_{i} F_{i}$, then $F \cup F_{i} \in \cup \mathscr{F}_{\alpha}(i=1, \cdots, n)$, and $F=\bigcap_{i}\left(F \cup F_{i}\right)$.

For any collection $\left\{G_{\alpha}\right\}$ of subgroups of a group $G$, we set $\wedge G_{\alpha}=$ $\cap G_{\alpha}$ and define $\vee G_{\alpha}$ as the smallest subgroup of $G$ which contains $G_{\alpha}$.

LEMMA 5. If $G$ is a subgroup of $\Pi(X)$ and $\left\{\mathscr{F}_{\alpha}\right\}_{\alpha \in A}$ is a collection of replete ecliptic families relative to $G$, then

$$
S\left(\wedge \mathscr{F}_{\alpha}, G\right)=\wedge S\left(\mathscr{F}_{\alpha}, G\right), S\left(\vee \mathscr{F}_{\alpha}, G\right) \supset \vee S\left(\mathscr{F}_{\alpha}, G\right)
$$

Proof. If $g \in S\left(\wedge \mathscr{F}_{\alpha}, G\right)$, then there is an $F \in \cap \mathscr{F}_{\alpha}$ such that $K(g) \supset F$, whence $g \in S\left(\mathscr{F}_{\alpha}, G\right)$ for each $\alpha \in A$. If $g \in \wedge S\left(\mathscr{F}_{\alpha}, G\right)$, then, for each $\alpha \in A$, there is an $F_{\alpha} \in \mathscr{F}_{\alpha}$ such that $K(g) \supset F_{\alpha}$. Hence, $K(g) \supset F=$ $\bigcup_{\alpha \in A} F_{\alpha}, F \in \mathscr{F}_{\beta}$ for each $\beta \in A$ since $\mathscr{F}_{\beta}$ is replete, and $g \in S\left(\wedge \mathscr{F}_{\alpha}, G\right)$. This proves the first relation. In the second, if $g \in \vee S\left(\mathscr{F}_{\alpha}, G\right)$, then there are sets $F_{1}, \cdots, F_{n} \in \bigcup_{\alpha \in A} \mathscr{F}_{\alpha}$ and elements $g_{1}, \cdots, g_{n} \in G$ such that $K\left(g_{i}\right) \supset F_{i}(i=1, \cdots, n)$ and $g=g_{1} \cdots g_{n}$. Hence, $K(g) \supset F=\bigcap_{i} F_{i}, F \in$ $\vee \mathscr{F}_{\alpha}$, and $g \in S\left(\vee \mathscr{F}_{\alpha}, G\right)$.

We now return to the case $X=B_{n}$.

Lemma 6. Let $\mathscr{G}$ be a family of (not necessarily closed) subsets of $S_{n-1}$ which

(a) satisfies (i), or

(b) is ecliptic relative to $H\left(B_{n}\right)$. Let $\mathscr{F}$ be the family of closed subsets of $B_{n}$ which contain a member of $\mathscr{G}$ in their interior (in the relative topology of $B_{n}$ ). Then

(a) $\mathscr{F}$ is ecliptic relative to $H_{0}\left(B_{n}\right)$, or

(b) $\mathscr{F}$ is ecliptic relative to $H\left(B_{n}\right)$. In either case, $\mathscr{F}$ is replete.

Proof. If $F_{0}, F_{0}^{\prime} \in \mathscr{G}$ and $F_{0} \subset \operatorname{int} F, F_{0}^{\prime} \subset \operatorname{int} F^{\prime}$, then $F_{0} \cap F_{0}^{\prime} \subset \operatorname{int}$ 
$F \cap \operatorname{int} F^{\prime}=\operatorname{int}\left(F \cap F^{\prime}\right)$, and $F \cap F^{\prime} \in \mathscr{F}$, whence $\mathscr{F}$ satisfies (i). In part (a), if $h \in H_{0}\left(B_{n}\right)$, then int $h(F)=h(\operatorname{int} F) \supset h\left(F_{0}\right)=F_{0}$, and $h(F) \in \mathscr{F}$. In part (b), if $h \in H\left(B_{n}\right)$, then there is an $F_{0}^{\prime \prime} \in \mathscr{C}$ such that $h\left(F_{0}\right) \supset F_{0}^{\prime \prime}$, and int $h(F) \supset F_{0}^{\prime \prime}$ as in (a), so that $h(F) \in \mathscr{F}$. Thus (ii) is verified in each case. The repleteness of $\mathscr{F}$ is obvious.

We will indicate the above relationship between $\mathscr{F}$ and $\mathscr{C}$ by saying that $\mathscr{F}$ is derived from $\mathscr{G}$. The simplest example of a derived ecliptic family relative to $H_{0}\left(B_{n}\right)$ is that in which $\mathscr{G}$ consists of a single subset of $S_{n-1}$. An ecliptic family relative to $H\left(B_{n}\right)$ is obtained by letting $\mathscr{G}$ consist of the complements in $S_{n-1}$ of finite subsets of $S_{n-1}$. When $n=2$, a family equivalent to the latter can be described as the set of complements in $B_{2}$ of interiors of simple closed curves which meet $S_{1}$ in a finite number of points. The construction can be varied by taking the set of complements of countable or first category subsets in $S_{n-1}$.

Returning to Lemma 5 and the case $X=B_{n}, G=H\left(B_{n}\right)$, we have not been able to determine whether equality holds in the second relation even for the case $S\left(\mathscr{F} \vee \mathscr{F}^{\prime}\right) \supset S(\mathscr{F}) \vee S\left(\mathscr{F}^{\prime}\right), \mathscr{F}^{-} \vee \mathscr{F}^{\prime}=\mathscr{C}(X)$. However, we do have the following result for derived families.

THEOREM 3. Let $\mathscr{F} \mathscr{F}^{\prime}$ be derived from $\mathscr{G}, \mathscr{C}^{\prime}$, respectively, where $\mathscr{G}=\left\{P_{0}\right\}, \mathscr{C}^{\prime}=\left\{Q_{0}\right\}$, and suppose that $\bar{P}_{0}, \bar{Q}_{0}$ can be separated in $S_{n-1}$ by an $(n-2)$-sphere $\sum \subset S_{n-1}$ which is tame relative to $H\left(B_{n}\right)$. Then

$$
S\left(\mathscr{F} \vee \mathscr{F}^{\prime}, H_{0}\left(B_{n}\right)\right)=S\left(\mathscr{F}, H_{0}\left(B_{n}\right)\right) \vee S\left(\mathscr{F}^{\prime}, H_{0}\left(B_{n}\right)\right)
$$

Proof. Let $I_{1}$ be an $(n-1)$-dimensional hyperplane passing through the center of $B_{n}$, and set $\Sigma_{1}=\Pi_{1} \cap S_{n-1}$. Choose $h \in H\left(B_{n}\right)$ such that $h(\Sigma)=\Sigma_{1}$. Since $\Pi_{1}$ and $h\left(\bar{Q}_{0}\right)$ are closed and disjoint, we can find a second hyperplane $\Pi_{2}$ parallel to $\Pi_{1}$ and lying between $\Pi_{1}$ and $h\left(\bar{Q}_{0}\right)$. Now $\Pi_{1}, \Pi_{2}$ divide $B_{n}$ into three regions (including boundaries) $\Delta_{1}, \Delta_{2}, \Delta_{2}$ such that $h\left(\bar{P}_{0}\right) \subset \Delta_{1}, h\left(\bar{Q}_{0}\right) \subset \Delta_{3}$. In fact, $P_{0} \subset \operatorname{int} h^{-1}\left(\Delta_{1}\right), Q_{0} \subset \operatorname{int} h^{-1}\left(\Delta_{3}\right)$, where int denotes interior in the relative topology of $B_{n}$. Hence, $h^{-1}\left(\Delta_{1}\right) \in \mathscr{F}$ and $h^{-1}\left(\Delta_{3}\right) \in \mathscr{F}^{\prime}$. Since these sets are disjoint, $\varnothing \in \mathscr{F} \vee \mathscr{F}^{\prime}$ and $S\left(\mathscr{F} \vee \mathscr{F}^{\prime}, H_{0}\left(B_{n}\right)\right)=H_{0}\left(B_{n}\right)$. By setting $\Delta_{1}=P, \Delta_{3}=Q$, and following the argument in the second half of the proof of Theorem 2, we can show that the group generated by those $g \in H_{0}\left(B_{n}\right)$ such that $K(g) \supset \Delta_{1}$ or $\Delta_{3}$ is precisely $H_{0}\left(B_{n}\right)$. Since $K(g) \supset \Delta_{1}$ implies $g \in S\left(h^{-1}(\mathscr{F}), H_{0}\left(B_{n}\right)\right)$, and $K(g) \supset \Delta_{3}$ implies $g \in S\left(h^{-1}\left(\mathscr{F}^{\prime}\right), H_{0}\left(B_{n}\right)\right)$, it follows from Lemma $2(c)$ that

$$
\begin{aligned}
H_{0}\left(B_{n}\right) & =h\left[S\left(h^{-1}(\mathscr{F}), H_{0}\left(B_{n}\right)\right) \vee S\left(h^{-1}\left(\mathscr{F}^{\prime}\right), H_{0}\left(B_{n}\right)\right)\right] h^{-1} \\
& =h\left[S\left(h^{-1}(\mathscr{F}), H_{0}\left(B_{n}\right)\right)\right] h^{-1} \vee h\left[S\left(h^{-1}\left(\mathscr{F}^{\prime}\right), H_{0}\left(B_{n}\right)\right)\right] h^{-1} \\
& =S\left(\mathscr{F}, H_{0}\left(B_{n}\right)\right) \vee S\left(\mathscr{F}^{\prime}, H_{0}\left(B_{n}\right)\right) .
\end{aligned}
$$


Hence, (2) is established. When $n=1$, the hypothesis of the theorem states that $P_{0}$ and $Q_{0}$ are the two points in $S_{0}$. The construction in the second half of the proof of Theorem 2 can evidently be carried through in this case.

6. Quotient spaces. we turn now to the problem of representing the quotient groups $H_{0}\left(B_{n}\right) / S(\mathscr{F})$, where $\mathscr{F}$ is an ecliptic family, as groups of transformations.

THEOREM 4. Let $A \subset S_{n-1}$ have the property that the set of its neighborhoods in $B_{n}$ has a countable base, and let $\mathscr{F}$ be the ecliptic family derived from $\{A\}$. Then $H_{0}\left(B_{n}\right) / S(\mathscr{F})$ can be represented as a group of order-preserving transformations of a partially ordered set $Z$ onto itself.

Proof. Let $Y$ be the set of all countable sequences $\left\{U_{k}\right\}$ of open subsets $U_{k} \subset B_{n}$ such that $U_{1} \supset U_{2} \supset \cdots$, and $\left\{U_{k}\right\}$ is a base for the set of neighborhoods of $A$. We introduce a partial ordering in $Y$ as follows: $\left\{U_{k}\right\} \leqq\left\{V_{k}\right\}$ if there exists a $k_{0}>0$ such that $k>k_{0}$ implies $U_{k} \subset V_{k}$. We call $\left\{U_{k}\right\}$ and $\left\{V_{k}\right\}$ equivalent if $\left\{U_{k}\right\} \leqq\left\{V_{k}\right\}$ and $\left\{V_{k}\right\} \leqq\left\{U_{k}\right\}$, and we write $\left\{U_{k}\right\} \equiv\left\{V_{k}\right\}$. Thus $\left\{U_{k}\right\} \equiv\left\{V_{k}\right\}$ means that $U_{k}=V_{k}$ for all but a finite set of $k$ 's. If $\left\{U_{k}\right\} \equiv\left\{V_{k}\right\}$ and $\left\{U_{k}\right\} \leqq\left\{W_{k}\right\}$, then clearly $\left\{V_{k}\right\} \leqq\left\{W_{k}\right\}$. Let $Z$ be the set of equivalence classes in $Y$ formed by the relation $\equiv$. If $u, v \in Z$, we define $u \leqq v$ to mean that the same ordering subsists between their respective equivalence classes. Moreover, $u \leqq v$ and $v \leqq u$ implies $u=v$.

If $h \in H_{0}\left(B_{n}\right)$ and $\left\{U_{k}\right\} \in Y$, then $\left\{h\left(U_{k}\right)\right\} \in Y$. Furthermore, $\left\{V_{k}\right\} \in Y$ and $\left\{U_{k}\right\} \leqq\left\{V_{k}\right\}$ implies $\left\{h\left(U_{k}\right)\right\} \leqq\left\{h\left(V_{k}\right)\right\}$. In particular, $\left\{U_{k}\right\} \equiv\left\{V_{k}\right\}$ implies $\left\{h\left(U_{k}\right)\right\} \equiv\left\{h\left(V_{k}\right)\right\}$. Thus, corresponding to $h$ there is an element $\omega(h) \in \Pi(Z)$ which is order-preserving, and $g \in H_{0}\left(B_{n}\right)$ implies $\omega(g h)=$ $\omega(g) \omega(h)$. We now show that $h \in S(\mathscr{F})$ if, and only if, $\omega(h)=i$, where $i$ is the identity in $\Pi(Z)$. If $h \in S(\mathscr{F})$, then there is an $F \subset B_{n}$ such that $K(h) \supset F$ and int $F \supset A$. For any $u \in Z$, let $\left\{U_{k}\right\}$ be a representative of $u$ in $Y$. Since $\left\{U_{k}\right\}$ is a base for the neighborhoods of $A$, we can find $k_{0}>0$ such that $k>k_{0}$ implies $U_{k} \subset \operatorname{int} F$, whence $\omega(h)(u)=u$, and $\omega(h)=i$. Conversely, if $h \notin S(\mathscr{F})$, then for each $\left\{U_{k}\right\} \in Y$, there exists a sequence $\left\{x_{k}\right\}$ of points in $B_{n}$ such that $x_{k} \in U_{k}$ and $h\left(x_{k}\right) \neq x_{k}(k=1$, $2, \cdots)$. Setting $V_{k}=U_{k} \cap c\left\{h\left(x_{k}\right)\right\}$ for each $k$, we have $\left\{V_{k}\right\} \in Y$ and $\left\{h\left(V_{k}\right)\right\} \not \equiv\left\{V_{k}\right\}$. If $\left\{V_{k}\right\}$ is a respresentative of $v \in Z$, then $\omega(h)(v) \neq v$, and $\omega(h) \neq i$. This proves our assertion. Let $\theta$ denote the canonical mapping from $H_{0}\left(B_{n}\right)$ onto $H_{0}\left(B_{n}\right) / S(\mathscr{F})$. Then $\theta(g)=\theta(h)$ if, and only if, $\omega(g)=\omega(h)$. Hence, $\omega \theta^{-1}$ is an isomorphism between $H_{0}\left(B_{n}\right) / S(\mathscr{F})$ and $\omega\left(H_{0}\left(B_{n}\right)\right)$.

If $A$ is closed in $B_{n}$, then $A$ is compact, and the uniform $(1 / k)$ - 
neighborhoods of $A$ form a base for its set of neighborhoods, so that the hypothesis of the theorem is satisfied in this case. If $A=S_{n-1}$, then $\mathscr{F}=\mathscr{F}_{0}$, and the construction in the proof allows us to represent $H\left(B_{n}\right) / S(\mathscr{F})$ as a subgroup of order-preserving elements in $\Pi(Z)$ which contains $\omega\left(H_{0}\left(B_{n}\right)\right)$.

\section{REFERENCES}

1. R. D. Anderson, The algebraic simplicity of certain groups of homeomorphisms, Amer. J. Math., 70 (1958), 955-963.

2. N. J. Fine and G. E. Schweigert, On the group of homeomorphisms of an arc, Annals of Math., 62 (1955), 237-253.

3. J. Schreier and S. Ulam, Eine Bemerkung uber die Gruppe der topologischen Abbildungen der Kreislinie auf sich selbst, Studia Math., 5 (1934), 155-159.

4. S. Ulam and J. von Neumann, On the group of homeomorphisms of the surface of the sphere, (abstract), Bull. Amer. Math. Soc., 53 (1947), 506.

UNIVERSITY OF BRITISH COLUMBIA 


\section{PACIFIC JOURNAL OF MATHEMATICS}

\section{EDITORS}

David GILbarg

Stanford University

Stanford, California

\section{F. H. BRowneLL}

University of Washington

Seattle 5, Washington

\section{A. L. Whiteman}

University of Southern California Los Angeles 7, California

\section{J. PAIGe}

University of California

Los Angeles 24, California

\section{ASSOCIATE EDITORS}
E. F. BECKENBACH
T. M. CHERRY
D. DERRY

\author{
E. HEWITT \\ A. HORN \\ L. NACHBIN
}

\author{
M. OHTSUKA \\ H. L. ROYDEN \\ M. M. SCHIFFER
}

E. SPANIER

E. G. STRAUS

F. WOLF

\section{SUPPORTING INSTITUTIONS}

\author{
UNIVERSITY OF BRITISH COLUMBIA \\ CALIFORNIA INSTITUTE OF TECHNOLOGY \\ UNIVERSITY OF CALIFORNIA \\ MONTANA STATE UNIVERSITY \\ UNIVERSITY OF NEVADA \\ NEW MEXICO STATE UNIVERSITY \\ OREGON STATE COLLEGE \\ UNIVERSITY OF OREGON \\ OSAKA UNIVERSITY \\ UNIVERSITY OF SOUTHERN CALIFORNIA
}

\author{
STANFORD UNIVERSITY \\ UNIVERSITY OF TOKYO \\ UNIVERSITY OF UTAH \\ WASHINGTON STATE COLLEGE \\ UNIVERSITY OF WASHINGTON \\ AMERICAN MATHEMATICAL SOCIETY \\ CALIFORNIA RESEARCH CORPORATION \\ HUGHES AIRCRAFT COMPANY \\ SPACE TECHNOLOGY LABORATORIES \\ NAVAL ORDNANCE TEST STATION
}

\footnotetext{
Mathematical papers intended for publication in the Pacific Journal of Mathematics should be typewritten (double spaced), and the author should keep a complete copy. Manuscripts may be sent to any one of the four editors. All other communications to the editors should be addressed to the managing editor, L. J. Paige at the University of California, Los Angeles 24, California.

50 reprints per author of each article are furnished free of charge; additional copies may be obtained at cost in multiples of 50 .
}

The Pacific Journal of Mathematics is published quarterly, in March, June, September, and December. The price per volume (4 numbers) is $\$ 12.00$; single issues, $\$ 3.50$. Back numbers are available. Special price to individual faculty members of supporting institutions and to individual members of the American Mathematical Society: $\$ 4.00$ per volume; single issues, $\$ 1.25$.

Subscriptions, orders for back numbers, and changes of address should be sent to Pacific Journal of Mathematics, 2120 Oxford Street, Berkeley 4, California.

Printed at Kokusai Bunken Insatsusha (International Academic Printing Co., Ltd.), No. 6, 2-chome, Fujimi-cho, Chiyoda-ku, Tokyo, Japan.

PUBLISHED BY PACIFIC JOURNAL OF MATHEMATICS, A NON-PROFIT CORPORATION

The Supporting Institutions listed above contribute to the cost of publication of this Journal, but they are not owners or publishers and have no responsibility for its content or policies. 


\section{Pacific Journal of Mathematics}

\section{Vol. 10, No. $4 \quad$ December, 1960}

M. Altman, An optimum cubically convergent iterative method of inverting a linear bounded operator in Hilbert space . . . . . . . . . . . . . . . . . . . . . . . . . . 1107

Nesmith Cornett Ankeny, Criterion for rth power residuacity ................. 1115

Julius Rubin Blum and David Lee Hanson, On invariant probability measures I . . . . . 1125

Frank Featherstone Bonsall, Positive operators compact in an auxiliary topology ..... 1131

Billy Joe Boyer, Summability of derived conjugate series . . . . . . . . . . . . . . . . 1139

Delmar L. Boyer, A note on a problem of Fuchs . . . . . . . . . . . . . . . . . 1147

Hans-Joachim Bremermann, The envelopes of holomorphy of tube domains in infinite

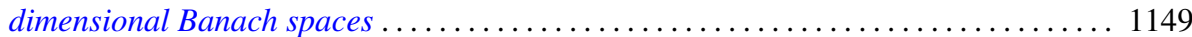

Andrew Michael Bruckner, Minimal superadditive extensions of superadditive

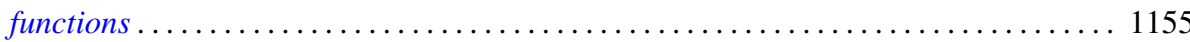

Billy Finney Bryant, On expansive homeomorphisms .................... 1163

Jean W. Butler, On complete and independent sets of operations in finite algebras . . . . . 1169

Lucien Le Cam, An approximation theorem for the Poisson binomial distribution ...... 1181

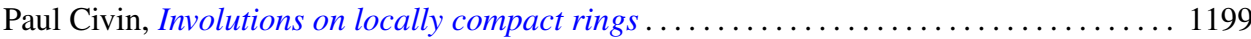

Earl A. Coddington, Normal extensions of formally normal operators . . . . . . . . . 1203

Jacob Feldman, Some classes of equivalent Gaussian processes on an interval ........ 1211

Shaul Foguel, Weak and strong convergence for Markov processes . . . . . . . . . . . 1221

Martin Fox, Some zero sum two-person games with moves in the unit interval ........ 1235

Robert Pertsch Gilbert, Singularities of three-dimensional harmonic functions . . . . . . . 1243

Branko Grünbaum, Partitions of mass-distributions and of convex bodies by

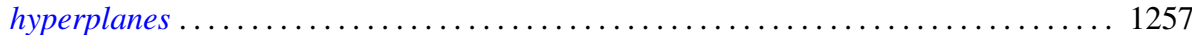

Sidney Morris Harmon, Regular covering surfaces of Riemann surfaces ........... 1263

Edwin Hewitt and Herbert S. Zuckerman, The multiplicative semigroup of integers

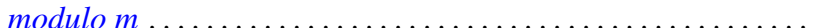

Paul Daniel Hill, Relation of a direct limit group to associated vector groups . ......... 1309

Calvin Virgil Holmes, Commutator groups of monomial groups . .

James Fredrik Jakobsen and W. R. Utz, The non-existence of expansive homeomorphisms

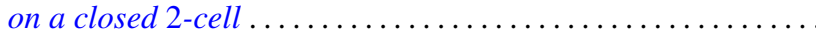

John William Jewett, Multiplication on classes of pseudo-analytic functions . . . . . . . 1323

Helmut Klingen, Analytic automorphisms of bounded symmetric complex domains . . . . 1327

Robert Jacob Koch, Ordered semigroups in partially ordered semigroups . . . . . . . . 1333

Marvin David Marcus and N. A. Khan, On a commutator result of Taussky and

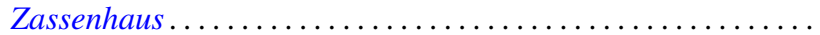

John Glen Marica and Steve Jerome Bryant, Unary algebras......

Edward Peter Merkes and W. T. Scott, On univalence of a continued fraction . . . . . . . 1361

Shu-Teh Chen Moy, Asymptotic properties of derivatives of stationary measures . . . . . 1371

John William Neuberger, Concerning boundary value problems . . . . . . . . . . . 1385

Edward C. Posner, Integral closure of differential rings . . . . . . . . . . . . . . . . . 1393

Marian Reichaw-Reichbach, Some theorems on mappings onto . . . . . . . . . . . . . 1397

Marvin Rosenblum and Harold Widom, Two extremal problems . . . . . . . . . . . . . . . . 1409

Morton Lincoln Slater and Herbert S. Wilf, A class of linear differential-difference

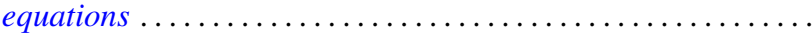

Charles Robson Storey, Jr., The structure of threads . . . . . . . . . . . . . . . . . . 1429

J. François Treves, An estimate for differential polynomials in $\partial / \partial z_{1},, \cdots, \partial / \partial z_{-} n \ldots \ldots 1447$

J. D. Weston, On the representation of operators by convolutions integrals . . . . . . . . 1453

James Victor Whittaker, Normal subgroups of some homeomorphism groups ......... 1469 